\title{
Role of the mTOR Pathway in Normal and Tumoral Adrenal Cells
}

\author{
M.C. De Martino a, b P.M. van Koetsveld ${ }^{a}$ R. Pivonello ${ }^{b}$ L.J. Hofland ${ }^{a}$ \\ a Department of Internal Medicine, Division of Endocrinology, Erasmus MC, Rotterdam, The Netherlands; \\ ${ }^{b}$ Department of Endocrinology and Oncology, 'Federico II' University, Naples, Italy
}

\section{Key Words}

mTOR $\cdot$ Insulin-like growth factor $\cdot$ Adrenocortical

carcinoma $\cdot$ Pheochromocytomas $\cdot$ mTOR inhibitor .

Rapamycin $\cdot$ Temsirolimus $\cdot$ Everolimus

\begin{abstract}
The mammalian target of rapamycin (mTOR) is a kinase of the phosphoinositide 3-kinase (PI3Ks)/protein kinase B (PKB or AKT) signaling pathway, which is one of the most important intracellular mediators of the activity of growth factors receptors, including vascular endothelial growth factor (VEGF) and insulin-like growth factors (IGFs). Dysregulation of the mTOR pathway has been found in many human tumors. Therefore, the mTOR pathway is considered as a target for antineoplastic therapy in several malignancies. Presently, the role and functions of mTOR and its signaling pathway in the normal and pathological adrenal gland has not been clarified yet. However, many growth factors and growth factor receptors, which are considered to play a role in the pathogenesis of adrenal tumors, can at least in part exert their effects through the activation of PI3K/AKT/mTOR pathway. Dysregulation of AKT has been reported in adrenocortical carcinomas and adrenomedullary tumors, named pheochromocytomas. Adrenocortical carcinomas and malignant pheochromocytomas are aggressive tumors with poor prognosis and scant treatment options. Therefore, new treatment options are warranted for these malignancies. On the
\end{abstract}

basis of the current knowledge, mTOR could play a role in the pathogenesis of both adrenocortical carcinomas and pheochromocytomas. Moreover, mTOR inhibitors, interfering with the activation of several mitogenic and angiogenic factors, could be considered as a novel treatment opportunity for the management of malignant adrenal tumors.

Copyright $\odot 2010$ S. Karger AG, Basel

\section{Introduction}

Adrenal tumors include benign and malignant cortical tumors and benign and malignant pheochromocytomas [1]. The malignant adrenal tumors are aggressive tumors with a poor prognosis [2-5]. The surgical removal of the tumor represents the treatment of choice for both benign and malignant hormone-secreting tumors, for all tumors suspected to be a pheochromocytoma, as well as for all non-hormone-secreting adrenal masses with clinical and radiological suspicion of malignancy $[1,2,6]$. However, in malignant tumors, surgery allows a complete remission only in a minority of cases with a diagnosis in the early stage of the disease $[3,4,6]$. The role of medical treatment in benign adrenocortical tumors is generally restricted to the treatment of the symptoms related to the hormonal hypersecretion using drugs interfering with the hormonal production and/or the hormonal actions (i.e. spironolactone, adrenolytic agents), in the time pre-

\section{KARGER}

Fax +4161306 1234

E-Mail karger@karger.ch

www.karger.com
(C) 2010 S. Karger AG, Basel

0028-3835/10/0925-0028\$26.00/0

Accessible online at:

www.karger.com/nen
Maria Cristina De Martino

Department of Internal Medicine, Division of Endocrinology, Room Ee 569

Erasmus Medical Center, Dr. Molewaterplein 50

NL-3015 GE Rotterdam (The Netherlands)

Tel. +31 10703 4394, Fax +31 10703 5430, E-Mail demartino.mc@gmail.com 
ceding the surgery. In adrenocortical carcinomas, the medical treatment with traditional cytotoxic chemotherapy is not satisfactory [5, 6]. Mitotane, the most commonly used adrenolytic agent, is able to control the hormonal hypersecretion in the majority of patients with hormonesecreting adrenocortical carcinomas, but has been associated with an objective tumor regression only in about $25 \%$ of cases [6]. In patients with pheochromocytomas anti-adrenergic agents (i.e. $\alpha$-adrenergic blockers) are used for the symptomatic treatment in the time preceding the surgery and to reduce the risks of adrenergic crisis during the surgery. In patients with inoperable malignant pheochromocytomas, besides the symptomatic treatment with antiadrenergic agents, radionuclide therapy with ${ }^{131}$ I-metaiodobenzylguanidine and combination chemotherapy, have been used. However, the results obtained with these treatments are still disappointing [3, $4,7]$.

The limited efficacy of conventional antineoplastic treatment in malignant adrenal tumors increases the need for novel, more effective treatment options. In recent years, the progress in understanding of the pathogenesis of tumors is encouraging and has initiated the development of the so-called 'targeted drugs', compounds that specifically interfere with molecular mechanisms involved in tumor cell growth and/or tumor vascular supply. Included in this category of drugs are compounds interfering with growth factor receptors (including proangiogenesis factors) and their related signaling pathways. Alterations of growth factors and their cognate receptors have been considered to be involved in the pathogenesis of both adrenocortical cancers and pheochromocytomas [7-9]. Moreover, tumor angiogenesis is essential for the growth and progression of solid tumors, including adrenal tumors $[7,8,10,11]$. Therefore, compounds interfering with tumor angiogenesis and growth factor signaling pathways represent a potential novel treatment opportunity for the management of malignant adrenal tumors.

\section{mTOR Pathway}

The mammalian target of rapamycin (mTOR) is a protein kinase of the phosphoinositide 3-kinase (PI3Ks)/ protein kinase $\mathrm{B}$ ( $\mathrm{PKB}$ or $\mathrm{AKT}$ ) signaling pathway, that forms multimolecular intracellular complexes and functions as a gatekeeper of metabolism as well as cell growth, receiving signals from sensors of cell stress, intracellular nutrients levels and several growth factors, including vas- cular endothelial growth factor (VEGF), insulin-like growth factors (IGFs), epidermal growth factor (EGF) and platelet-derived growth factor (PDGF) [12-15]. The complexes formed by mTOR are named mTORC1 and mTORC2, and are sensitive or insensitive to rapamycin, respectively, the oldest compound among the MTOR inhibitors [16]. The binding of many growth factors to their cognate receptor tyrosine kinases (RTKs) leads to the recruitment of PI3K to the membrane, where this enzyme converts phosphatidylinositol bisphosphate (PIP2) to phosphatidylinositol trisphosphate (PIP3), providing docking sites for signaling proteins, including 3-phosphoinositide-dependent protein kinase 1 (PDK1) and the serine-threonine kinase AKT. These events are antagonized by PTEN (phosphatase and tensin homologue) that limits the growth factor signaling by dephosphorylating PIP3. Figure 1 shows a simplified scheme of growth factor-induced activation of the mTOR pathway and the sites of action of compounds acting as mTOR inhibitors. PDK1 is a serine-threonine kinase that phosphorylates and activates AKT, which elicits many downstream signaling events including the activation of mTORC1. AKT promotes mTORC1 activity mainly by phosphorylating the tuberosus-sclerosis complex (TSC1/2) $[16,17]$. This phosphorylation leads to the inactivation of TSC2 that is an indirect inhibitor of $\mathrm{mTORC} 1$ and results in the activation of this complex [16]. mTOR as part of mTORC1 complex can also be directly phosphorylated by AKT, forming phospho-mTOR (p-mTOR), that is often studied as a marker of mTOR activation. However, whether this phosphorylation is crucial or not in the activation of the mTORC1 complex is still a matter of discussion [16, 17]. Activation of the mTORC1 complex leads to the phosphorylation and activation of downstream effectors of the pathway: the protein kinase p70 ribosomal protein S6 kinase 1 (S6K1) and eukaryotic translation initiation factor 4E-binding proteins (4EBP1) [16]. S6K1 and 4EBP1 are both regulators of mRNA translation and stimulate the synthesis of several oncogenic proteins such us c-Myc, hypoxia-inducible factor-1 $\alpha$ (HIF-1 $\alpha)$, VEGF, IGF-II and cyclin D [13]. Conversely, mTORC2 regulates the cytoskeleton function and it has been proposed to be an important activator of the AKT function. Growth factors stimulate mTORC2, activity and some components of mTORC2 complex are phosphorylated as consequences of growth factor stimulation, but the responsible kinases are uncertain [16]. Therefore, there is evidence that mTOR as part of both complexes (mTORC1 and mTORC2) mediates the intracellular signaling induced by growth factor receptor activation (fig. 1). However, mTORC1 is 
Fig. 1. Simplified scheme of growth factorinduced activation of the mTOR pathway and actions of mTOR inhibitors.

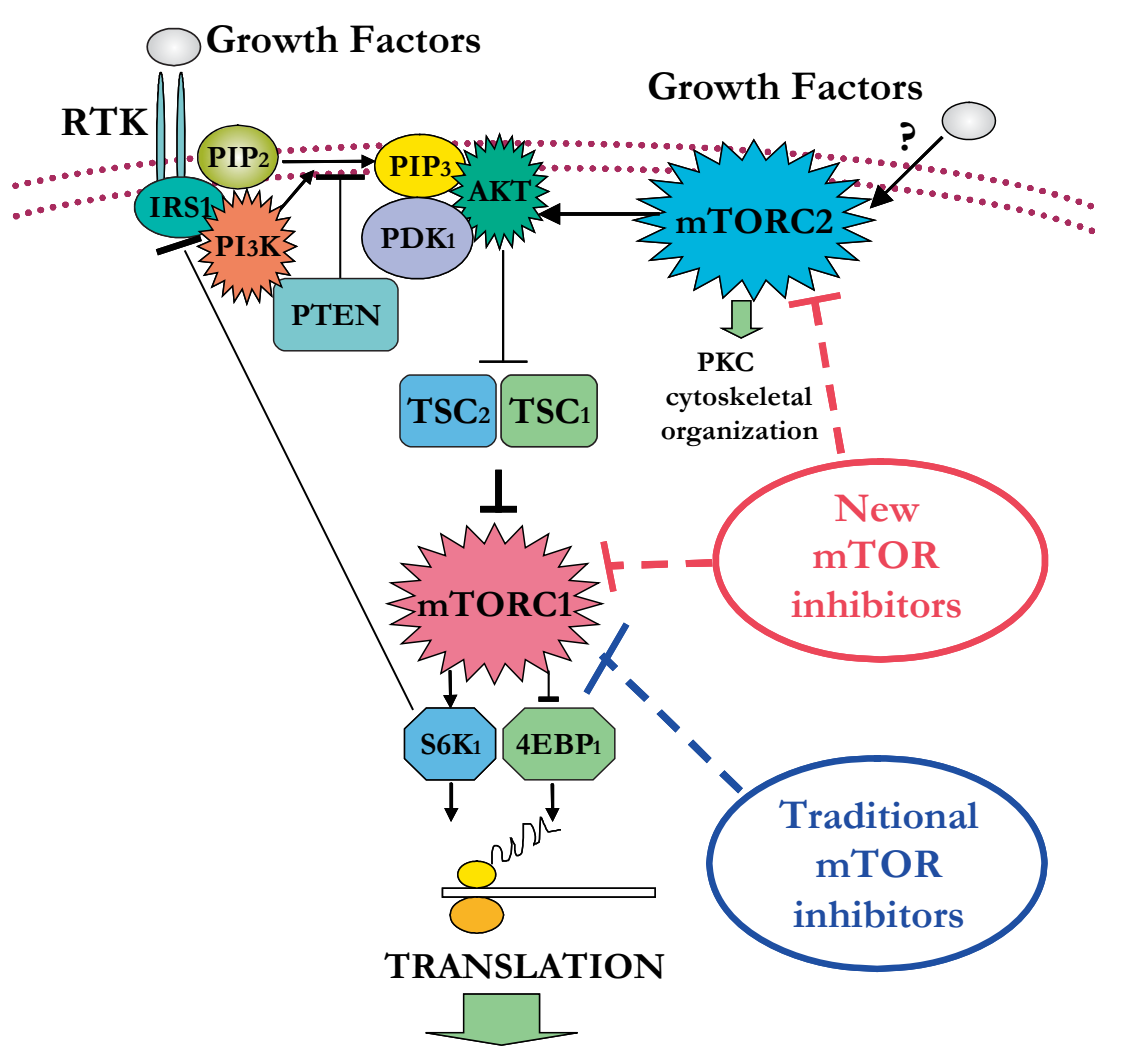

GROWTH-PROLIFERATION-SURVIVAL-ANGIOGENESIS downstream to AKT and mTORC2 seems to be upstream to $\mathrm{AKT}[13,16]$.

It is noteworthy that mTOR is an intracellular mediator of IGFII and VEGF activity and, in turn, is able to control the synthesis of these growth factors [13]. VEGF is one of the most important mediators of angiogenesis in solid tumors including adrenal tumors. Moreover, growth factors such as IGFs and VEGF are autocrine/paracrine regulators of tumoral cell growth in many different types of tumors, including adrenal tumors (fig. 2) [8-11, 18]. Dysregulation of the mTOR pathway has been found in many human tumors, including neuroendocrine tumors $[13,14,19,20]$. Therefore, the mTOR pathway is considered an important target for antineoplastic therapies and it is conceivable that it could also play a role in the pathogenesis of adrenal tumors. mTOR inhibitors may exert their antitumor effects indirectly, by inhibiting tumor angiogenesis, and directly by inhibiting cell growth and proliferation [16]. It is conceivable that mTOR inhibitors, interfering with several mitogenic and angiogenic factors, could inhibit adrenal tumor growth.

Presently, many clinical trials (phase I-III) are investigating the antineoplastic effects of mTOR inhibitors, mainly targeting the mTORC1 complex (shown in fig. 1 as 'traditional' mTOR inhibitors), such as sirolimus (rapamycin), temsirolimus (CCI-779), everolimus (RAD001) and deforolimus (AP23573), alone or in combination with other compounds, in several kinds of solid tumors and hematological malignancies. On the basis of the clinical studies already concluded, a safe schedule of treatment has been defined for each of these compounds and promising results of antitumor activity have been achieved in a variety of malignancies, and some of these compounds are already approved for the treatment of advanced renal cell carcinoma $[13,14,17,19]$.

The activity of mTORC 2 is not directly inhibited by the above-listed compounds, but it has been reported that long-term treatment with rapamycin can inhibit the activ- 
ity of mTORC2 by the sequestering of mTOR as part of the mTORC1 complex [13]. However, new compounds, able to directly inhibit both mTORC complexes such us AZD8055 and OSI-027 (reported in fig. 1 as 'new' mTOR inhibitors) are proceeding in the early clinical testing [17]. The results of these studies will answer the question whether the use of compounds blocking both mTORC complexes could have additional advantage over the use of traditional mTOR inhibitors in the treatment of tumors.

To the best of our knowledge, there are no preclinical or clinical studies evaluating the effects of mTOR inhibitor drugs in adrenocortical tumors and only very limited data in pheochromocytomas [20-22].

\section{Role of the mTOR Pathway in Normal Adrenal Gland}

Up to our knowledge, there are no studies evaluating the mTOR pathway in the normal adrenal gland. The expression of mTOR, p-mTOR, AKT and phospho-AKT (p$\mathrm{AKT}$ ) in the normal adrenal gland has been evaluated only as a control for adrenal tumors [20,23]. In 3 out of 3 normal adrenals, no staining for p-mTOR and p-AKT, evaluated by immunohistochemistry, has been reported in the normal adrenal medullas [23]. Fassnacht et al. [20] have evaluated the expression of total-AKT and p-AKT in 4 normal adrenal glands by immunohistochemistry. They described a notable staining for total-AKT and p$\mathrm{AKT}$ in the reticularis layer, whereas a weaker expression of total- and p-AKT was found in the fasciculata and glomerulosa and only a faint staining for total-AKT in the normal medulla. These preliminary observations suggest that there is a layer-specific pattern of activation of the PI3Ks/AKT/mTOR pathway in the normal adrenal gland, which seems to be predominantly inactive in the normal adrenal medulla and active in the reticularis layer of the adrenal cortex. IGFs have been reported to stimulate adrenal steroidogenesis, particularly androgen production [18]. The activation of PI3K/AKT/mTOR pathway in the reticularis of normal adrenals suggests a potential role of this pathway in the control of androgen production, probably as intracellular mediator of the IGFs.

\section{Potential Role of the mTOR Pathway in Adrenal Tumors}

Presently, the role and function of mTOR and its pathway in adrenal tumors have not yet been clarified. However, total-AKT has been found to be overexpressed both in adrenocortical carcinomas and in pheochromocytomas, compared to normal adrenals or adenomas [20]. The corresponding activated form, $\mathrm{p}$-AKT, has also been reported to be overexpressed in pheochromocytomas [20, 24]. Equivocal data exist with the respect to p-AKT expression in adrenocortical carcinomas [20, 25]. Indeed, some authors report overexpression of $\mathrm{p}-\mathrm{AKT}$ in adrenocortical carcinomas [25], while others do not [20]. Mice with a heterozygous deletion of the PTEN gene present an increased tendency to develop pheochromocytomas [26]. However, PTEN mutations appear to be rare in human pheochromocytomas $[27,28]$, suggesting that in human pheochromocytomas the mechanisms involved in the overactivation of $\mathrm{p}-\mathrm{AKT}$ are different from mice.

In H295 and SW13, two different human adrenocortical carcinoma cell lines [25, 29], and in PC12, a rat pheochromocytoma cell line [30], IGF-I stimulation has been correlated with increased AKT phosphorylation, suggesting that AKT is one of the intracellular mediators of IGF signaling in adrenal tumors. In PC12 cells, the effects of some growth factors on downstream components of the mTOR pathway have also been evaluated. EGF and nerve growth factor (NGF) were reported to stimulate the phosphorylation of 4EBP1 [31] and EGF has also been shown to increase the phosphorylation of S6K1 [32]. These effects were prevented by the administration of rapamycin [32].

As indicated above, it has been shown that mTOR can stimulate the translation of IGF-II and VEGF. In turn, this stimulation can be induced by the same growth factors [13]. Therefore, mTOR could be one of the intracellular mediators of the pro-survival/pro-growth effects of growth factor signaling in adrenal tumors and it could be an intracellular component to the pro-growth autocrine loops and pro-angiogenetic events, considered to be involved in the pathogenesis of adrenal tumors, as shown in figure 2 .

\section{Role of mTOR as a Drug Target in the Treatment of Adrenal Tumors}

To the best of our knowledge, there are no clinical or preclinical published data evaluating the effects of mTOR inhibitors in adrenocortical carcinomas. However, compounds blocking IGF-I receptor activity have been found to inhibit the proliferation of $\mathrm{H} 295$ cells $[25,33]$. These effects have been correlated with a reduction of p-AKT [25], suggesting that AKT itself and/or mTOR could be a potential target for the treatment of adrenocortical car- 
Fig. 2. Potential role of the mTOR pathway in adrenal tumors.

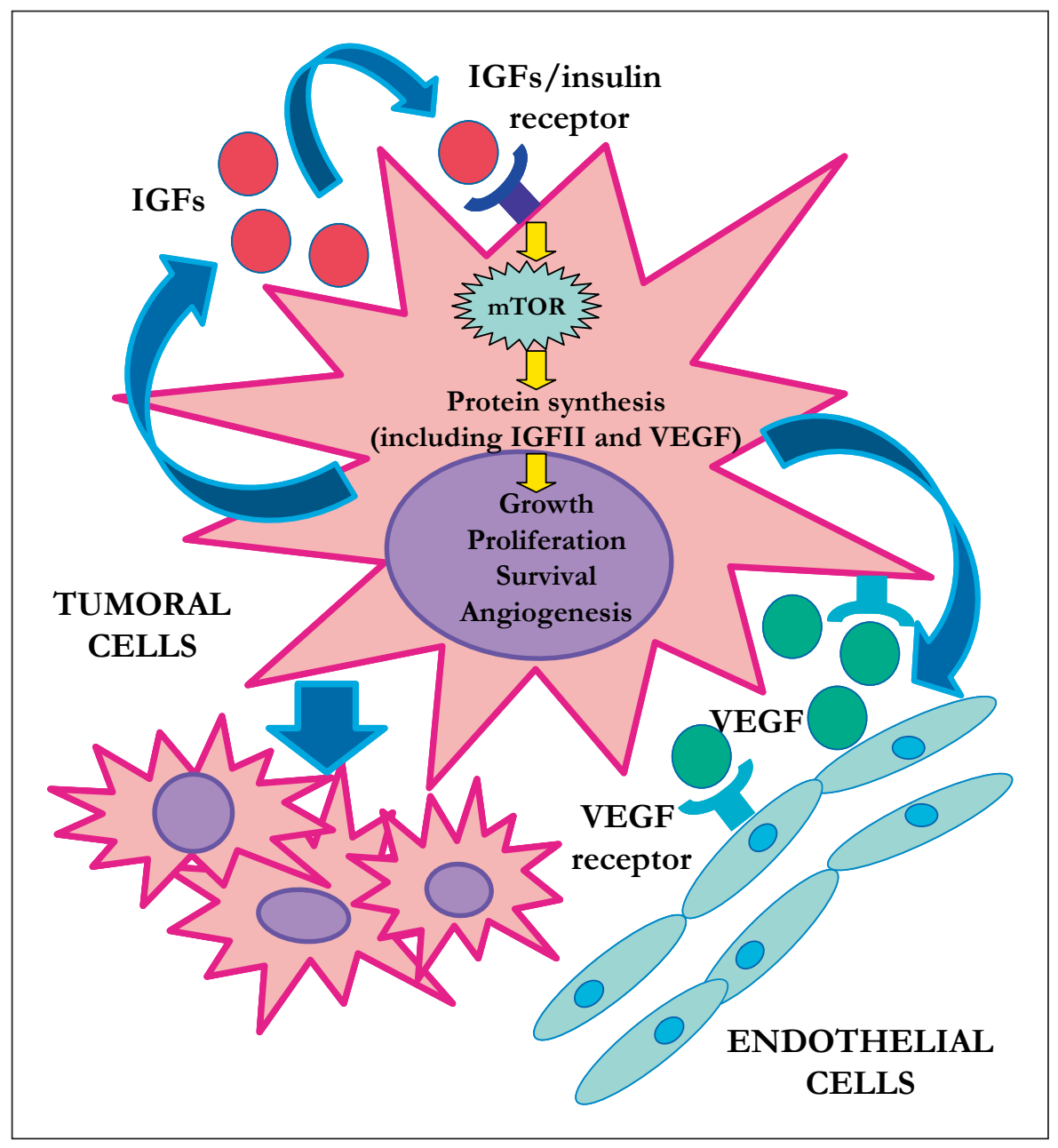

cinomas. Inhibition of the IGF-I receptor in preclinical models of human adrenocortical carcinomas has been associated with a reduction of VEGF production [25]. According with the described role of mTOR in VEGF production [13], it is conceivable that the inhibition of VEGF induced by the IGF-I receptor inhibitors is related to the inhibition of the mTOR pathway. LY294002, an AKT inhibitor, has been found to inhibit proliferation of PC12 cells in a time- and dose-dependent manner [24]. In neuroblastoma cell lines (a tumor closely related to pheochromocytoma), rapamycin inhibits cell proliferation by inducing cell cycle arrest [34]. Rapamycin, evaluated as a single dosage, has been found to significantly inhibit cell growth in the normal chromaffin rat cells, but not in the PC12 cell line [22]. In a recent study evaluating the effects of glucacon-like-peptide-1 (GLP-1) in PC12 cells, used as a model for neuronal cells, the authors show that GLP-1, through the activation of PI3K/AKT/mTOR pathway, is able to protect these cells from oxidative stress-induced cell apoptosis [35]. These protective effects were inhibited by the treatment with rapamycin. In an animal model for pheochromocytoma, temsirolimus was able to inhibit tumor progression [26]. This effect could either be attributed to a direct effect of the mTOR inhibitor on tumoral cell proliferation or to the inhibitory effects of temsirolimus on vascular growth. Therefore, further preclinical studies should be performed to clarify whether these compounds might have a direct effect on pheochromocytoma cells, whether these effects could depend on the presence of growth factors in the cell environment, and whether the combination treatment using mTOR inhibitors and other compounds, such us conventional chemotherapics or other targeted drugs (i.e. IGF-I receptor antagonists), could have stronger antineoplastic effects than the monotherapy. The promising effects of mTOR inhibitors in the treatment of neuroendocrine tumors $[36,37]$ 
have encouraged the use of mTOR inhibitors in the treatment of some patients with advanced malignant pheochromocytomas [21]. Unfortunately, in these 4 cases the treatment did not produce promising results. However, all these patients started treatment in a very late stage of disease; therefore, we cannot exclude that the use of mTOR inhibitors in an earlier stage of disease could have more beneficial effects.

\section{Conclusion and Future Direction}

In conclusion, the PI3Ks/AKT/mTOR pathway could be one of the intracellular mediators of growth factors, both in normal adrenal (particularly in the reticularis layer of the adrenal cortex) and in adrenal tumors. Moreover, this pathway could be involved in the pathogenesis of adrenal tumors and it could play a role in adrenal tumor angiogenesis. Therefore, the PI3Ks/AKT/mTOR pathway could be a potential target for the treatment of adrenal malignancies. However, there is still a lack of preclinical studies that address the effects of mTOR inhibitors in models for adrenal tumors. To the best of our knowledge, there are no data regarding the effects of new mTOR inhibitors (inhibiting both mTORC complexes) or with the respect to the use of MTOR inhibitors in combination with other compounds (such as drugs targeting the IGF or VEGF system, somatostatin analogs, mitogenactivated protein kinase (MAPK) inhibitors or cytotoxic compounds (including mitotane for adrenocortical carcinomas) in adrenal tumors. Therefore, studies are clearly warranted.

\section{Disclosure Statement}

The authors have nothing to disclose.

\section{References}

1 Latronico AC, Chrousos GP: Extensive personal experience: adrenocortical tumors. J Clin Endocrinol Metab 1997;82:1317-1324.

-2 Kirschner LS: Emerging treatment strategies for adrenocortical carcinoma: a new hope. J Clin Endocrinol Metab 2006;91:14-21.

3 Pacak K, Eisenhofer G, Ahlman H, Bornstein SR, Gimenez-Roqueplo AP, Grossman AB, Kimura N, Mannelli M, McNicol AM, Tischler AS: Pheochromocytoma: recommendations for clinical practice from the First International Symposium. October 2005. Nat Clin Pract 2007;3:92-102.

-4 Lenders JW, Eisenhofer G, Mannelli M, Pacak K: Phaeochromocytoma. Lancet 2005; 366:665-675

5 Fassnacht M, Allolio B: Clinical management of adrenocortical carcinoma. Best Prac Res 2009;23:273-289.

-6 Allolio B, Fassnacht M: Clinical review: adrenocortical carcinoma: clinical update. J Clin Endocrinol Metab 2006;91:2027-2037.

-7 Fassnacht M, Kreissl MC, Weismann D, Allolio B: New targets and therapeutic approaches for endocrine malignancies. Pharmacol Ther 2009;123:117-141.

-8 Volante M, Buttigliero C, Greco E, Berruti A, Papotti M: Pathological and molecular features of adrenocortical carcinoma: an update. J Clin Pathol 2008;61:787-793.

9 Fottner C, Minnemann T, Kalmbach S, Weber MM: Overexpression of the insulin-like growth factor I receptor in human pheochromocytomas. J Mol Endocrinol 2006;36: 279-287.
10 Takekoshi K, Isobe K, Yashiro T, Hara $\mathrm{H}$, Ishii K, Kawakami Y, Nakai T, Okuda Y: Expression of vascular endothelial growth factor (VEGF) and its cognate receptors in human pheochromocytomas. Life Sci 2004;74: 863-871.

11 Salmenkivi K, Heikkila P, Liu J, Haglund C, Arola J: VEGF in 105 pheochromocytomas: enhanced expression correlates with malignant outcome. Apmis 2003;111:458-464.

12 Hanna SC, Heathcote SA, Kim WY: mTOR pathway in renal cell carcinoma. Expert Rey Anticancer Ther 2008;8:283-292.

13 Wan X, Helman LJ: The biology behind mTOR inhibition in sarcoma. Oncologist 2007;12:1007-1018.

14 Konings IR, Verweij J, Wiemer EA, Sleijfer S: The applicability of mTOR inhibition in solid tumors. Curr Cancer Drug Targets 2009; 9:439-450

$\checkmark 15$ Le Tourneau C, Faivre S, Serova M, Raymond E: mTORC1 inhibitors: is temsirolimus in renal cancer telling us how they really work? Br J Cancer 2008;99:1197-1203.

16 Guertin DA, Sabatini DM: Defining the role of mTOR in cancer. Cancer Cell 2007;12:922.

17 Liu P, Cheng H, Roberts TM, Zhao JJ: Targeting the phosphoinositide 3-kinase pathway in cancer. Nat Rev 2009;8:627-644.

18 Fottner C, Hoeflich A, Wolf E, Weber MM: Role of the insulin-like growth factor system in adrenocortical growth control and carcinogenesis. Horm Metab Res 2004;36:397405 .
19 LoPiccolo J, Blumenthal GM, Bernstein WB, Dennis PA: Targeting the PI3K/Akt/mTOR pathway: effective combinations and clinical considerations. Drug Resist Updat 2008;11: 32-50.

20 Fassnacht M, Weismann D, Ebert S, Adam P, Zink M, Beuschlein F, Hahner S, Allolio B: AKT is highly phosphorylated in pheochromocytomas but not in benign adrenocortical tumors. J Clin Endocrinol Metab 2005;90: 4366-4370.

-21 Druce MR, Kaltsas GA, Fraenkel M, Gross DJ, Grossman AB: Novel and evolving therapies in the treatment of malignant phaeochromocytoma: experience with the mTOR inhibitor everolimus (RAD001). Horm Metabol Res 2009;41:697-702.

22 Powers JF, Tischler AS, Cherington V: Discordant effects of rapamycin on proliferation and p70S6 kinase phosphorylation in normal and neoplastic rat chromaffin cells. Neurosci Lett 1999;259:137-140.

-23 Johnsen JI, Segerstrom L, Orrego A, Elfman L, Henriksson M, Kagedal B, Eksborg S, Sveinbjornsson B, Kogner P: Inhibitors of mammalian target of rapamycin downregulate MYCN protein expression and inhibit neuroblastoma growth in vitro and in vivo. Oncogene 2008;27:2910-2922.

-24 Adler JT, Hottinger DG, Kunnimalaiyaan $\mathrm{M}$, Chen $\mathrm{H}$ : Inhibition of the PI3K pathway suppresses hormonal secretion and limits growth in pheochromocytoma cells. Wld J Surg 2009;33:2452-2457. 
-25 Barlaskar FM, Spalding AC, Heaton JH, Kuick R, Kim AC, Thomas DG, Giordano TJ, Ben-Josef E, Hammer GD: Preclinical targeting of the type I insulin-like growth factor receptor in adrenocortical carcinoma. J Clin Endocrinol Metab 2009;94:204-212.

-26 Podsypanina K, Lee RT, Politis C, Hennessy I, Crane A, Puc J, Neshat M, Wang H, Yang L, Gibbons J, Frost P, Dreisbach V, Blenis J, Gaciong Z, Fisher P, Sawyers C, Hedrick-Ellenson L, Parsons R: An inhibitor of mTOR reduces neoplasia and normalizes $\mathrm{p} 70 / \mathrm{S} 6 \mathrm{ki}-$ nase activity in Pten+/- mice. Proc Natl Acad Sci USA 2001;98:10320-10325.

-27 van Nederveen FH, Perren A, Dannenberg H, Petri BJ, Dinjens WN, Komminoth P, de Krijger RR: PTEN gene loss, but not mutation, in benign and malignant phaeochromocytomas. J Pathol 2006;209:274-280.

-28 Puc J, Placha G, Wocial B, Podsypanina K, Parsons R, Gaciong Z: Analysis of PTEN mutation in non-familial pheochromocytoma. Ann NY Acad Sci 2006;1073:317-331.
29 Cantini G, Lombardi A, Piscitelli E, Poli G, Ceni E, Marchiani S, Ercolino T, Galli A, Serio M, Mannelli M, Luconi M: Rosiglitazone inhibits adrenocortical cancer cell proliferation by interfering with the IGF-IR intracellular signaling. PPAR Res 2008;2008:904041.

-30 Lu X, Kambe F, Cao X, Yamauchi M, Seo H: Insulin-like growth factor-I activation of Akt survival cascade in neuronal cells requires the presence of its cognate receptor in caveolae. Exp Cell Res 2008;314:342-351.

31 Kleijn M, Korthout MM, Voorma HO, Thomas AA: Phosphorylation of the eIF4Ebinding protein PHAS-I after exposure of PC12 cells to EGF and NGF. FEBS Letters 1996;396:165-171.

32 Harthill JE, Pozuelo Rubio M, Milne FC, MacKintosh C: Regulation of the 14-3-3binding protein p39 by growth factors and nutrients in rat PC12 pheochromocytoma cells. Biochem J 2002;368:565-572.

33 Almeida MQ, Fragoso MC, Lotfi CF, Santos MG, Nishi MY, Costa MH, Lerario AM, Maciel CC, Mattos GE, Jorge AA, Mendonca BB, Latronico AC: Expression of insulin-like growth factor-II and its receptor in pediatric and adult adrenocortical tumors. J Clin Endocrinol Metabol 2008;93:3524-3531.

34 Misawa A, Hosoi H, Tsuchiya K, Sugimoto T: Rapamycin inhibits proliferation of human neuroblastoma cells without suppression of MycN. Int J Cancer 2003;104:233-237.
35 Kimura R, Okouchi M, Fujioka H, Ichiyanagi $A$, Ryuge $F$, Mizuno $T$, Imaeda $K$, Okayama N, Kamiya Y, Asai K, Joh T: Glucagon-like peptide-1 (GLP-1) protects against methylglyoxal-induced PC12 cell apoptosis through the PI3K/Akt/mTOR/GCLc/redox signaling pathway. Neuroscience 2009;162: 1212-1219.

36 Yao JC, Lombard-Bohas C, Baudin E, Kvols LK, Rougier P, Ruszniewski P, Hoosen S, St Peter J, Haas T, Lebwohl D, Van Cutsem E, Kulke MH, Hobday TJ, O'Dorisio TM, Shah MH, Cadiot G, Luppi G, Posey JA, Wiedenmann B: Daily oral everolimus activity in patients with metastatic pancreatic neuroendocrine tumors after failure of cytotoxic chemotherapy: a phase II trial. J Clin Oncol 2010;28:69-76.

37 Yao JC, Phan AT, Chang DZ, Wolff RA, Hess K, Gupta S, Jacobs C, Mares JE, Landgraf AN, Rashid A, Meric-Bernstam F: Efficacy of RAD001 (everolimus) and octreotide LAR in advanced low- to intermediate-grade neuroendocrine tumors: results of a phase II study. J Clin Oncol 2008;26:4311-4318. 